\title{
Histological Changes of Selected Westar Rat Tissues Following the Ingestion of Tartrazine With Special Emphasis on the Protective Effect of Royal Jelly and Cod Liveroil
}

\author{
Wael AM Ghonimi* and Ahmad Elbaz
}

Department of Histology and Cytology, Faculty of Veterinary Medicine, Zagazig University, Zagazig, Egypt

\begin{abstract}
The present investigation was carried out on thirty six mature Westar rats from laboratory animal unit in the faculty of veterinary medicine, Zagazig University, Egypt. The animals were divided into six groups of six animals for each. Group I was considered as a control group. Group II was treated with tartrazine only in a dose $500 \mathrm{mg} / \mathrm{kg} \mathrm{body}$ weight by oral gavage for 30 day. Group III was treated with the same dose of tartrazine with $300 \mathrm{mg} / \mathrm{kg}$ body weight Royal jelly. Group IV was treated with tartrazine and Code liver oil in a dose $0.4 \mathrm{mg} / \mathrm{kg}$ body weight. While the last two groups, V and VI were treated with Code liver oil and Royal jelly separately by the above mentioned doses for 30 days. At the end of experiment, cervical dislocation of rats and for the histological studies, the liver, kidney, testis and brain tissues in addition to stomach were separated and small pieces from them were taken, fixed in neutral buffered formalin $10 \%$, dehydrated, cleared and processed for paraffin blocks and 5 micron sections were obtained, mounted on a glass slides and stained histologically with Hematoxylin and Eosin (H\&E) and Blue Masson's Trichrome. Groups I, V, VI showed normal histological structure of all selected tissues without any abnormalities. Group II showed severe steatosis, diffuse degeneration, necrosis of hepatic tissues, fibrous tissue proliferation with anti-inflammatory cells infiltration in the portal areas and moderate disorganization of hepatic cords. Furthermore, hyperplasia of the interstitial connective tissue and Leydig cells with marked vacuolations were recognized. Moreover, slightly vacuolations in brain tissues were recognized specially within the white matter as well as degenerative changes in stomach mucosa. And also, hyaline degeneration in renal tubules and vacuolations of glomeruli were observed. The present investigation concluded that the serious effect of tartrazine toxicity was more pronounced in liver then kidney, stomach, testis and brain tissues respectively. Moreover, the curative protective effect of both royal jelly and cod liver oil are non-significant against tartrazine toxicity.
\end{abstract}

Keywords: Tartrazine; Histoarchitecture; Westar Rat; Steatosis; Hyperplasia; Vacuolation; Necrosis; Degeneration

\section{Introduction}

Food additives play a vital role in food supply industries, they allow growing population of the world to enjoy a variety of safe, wholesome, and tasty foods. food cookers are materials of natural origin have been used to provide color in food,drugs and cosmetics for a thousands of years. By the early 1995, natural and synthetic colors additives were used extensively to color the foods, drugs and cosmetics [1]. The most famous food additive which is used as a coloring substance is tartrazine. Tartrazine is known as E102 or FD and C, yellow 5 and characterized by its lemon yellow azo dye, and water soluble. Many products contain tartrazine like foods cotton candy, soft drinks, flavored chips (Doritos, Nachos, etc.), cereals (corn lakes, muesli, etc.), cake mixes, soups, sauces, some rice, ice cream, candy, chewing gum, marzipan, jam and jelly, some of non-food products include tartrazine such as soaps, cosmetics, shampoos and other hair products, also some medical preparations contain tartrazine such as vitamins, antacids, medicinal capsules and certain prescription drugs. The acceptable daily intake (ADI) for humans is $7.5 \mathrm{mg} / \mathrm{kg}$ body weight/day [2]. Tartrazine has been implicated as the food additive which is most often responsible for allergic reactions in specific human. When the Tartrazine reaches the intestine, it can undergo metabolic reduction by intestinal micro flora [3], and the reductive cleavage products are rapidly absorbed. Following reductive cleavage of the azo linkage by intestinal bacteria, Sulfanilic acid and aminopyrazolone are produced. The pyrazolone fragment is further degraded by intestinal bacteria to yield a second molecule of Sulfanilic acid [4].

Royal jelly (RJ) is a part of diet of honey bee larvae of great antioxidant and immunostimulant properties, and it contains many important compounds with biological activity, such as free amino acids, proteins, sugars, fatty acids (mainly 10-hydroxy-2-decenoic acid; 10-HDA), minerals (mainly iron and calcium) and vitamins (mainly thiamine, niacin, riboflavin) $[5,6]$. And also, cod liver oil (CLO) has a potent antioxidant effect against much oxidative stress as diabetes and toxicity [7].

\section{Aim of Work}

The present study was performed to investigate the sub-chronic toxicity of tartrazine as well as the protective effects of both royal jelly and cod liver oil in some visceral organs in Westar rats.

\section{Materials and Methods}

\section{Chemicals}

Tartrazine (CAS 1934-21-0, Purity 86.7\%), was purchased from

*Corresponding author: Wael AM Ghonimi, Department of Histology and Cytology, Faculty of Veterinary Medicine, Zagazig University, Zagazig, Egypt, Tel: 00201222498246; Fax: +2-055-2283683; E-mail: drwael_histology@yahoo.com; waghonimi@zu.edu.eg

Received June 06, 2015; Accepted July 10, 2015; Published July 12, 2015.

Citation: Ghonimi WAM, Elbaz A (2015) Histological Changes of Selected Westar Rat Tissues Following the Ingestion of Tartrazine With Special Emphasis on the Protective Effect of Royal Jelly and Cod Liveroil. J Cytol Histol 6: 346. doi:10.4172/2157-7099.1000346

Copyright: $\odot 2015$ Ghonimi WAM, et al. This is an open-access article distributed under the terms of the Creative Commons Attribution License, which permits unrestricted use, distribution, and reproduction in any medium, provided the original author and source are credited. 
Citation: Ghonimi WAM, Elbaz A (2015) Histological Changes of Selected Westar Rat Tissues Following the Ingestion of Tartrazine With Special Emphasis on the Protective Effect of Royal Jelly and Cod Liveroil. J Cytol Histol 6: 346. doi:10.4172/2157-7099.1000346

Alfa Aesar, Sigma (Germany). Royal jelly produced by honey bees as well as cod liver oil was purchased from pharmacy in Zagazig, Egypt.

\section{Animals and housing}

Westar rats weighing between 170 and $200 \mathrm{~g}$ were housed in a controlled room with suitable conditions as normal temperature and light dark cycle. They were kept in transparent polypropylene cages with free access to water and dry rat pellets feeds.

\section{Experimental design}

The animals were divided into six groups of six animals each. Group I was considered as a control group, food and water only. Group II was obtained a tartrazine only in a dose $500 \mathrm{mg} / \mathrm{kg}$ body weight by oral gavage for 30 day. Group III was obtained the same dose of tartrazine with $300 \mathrm{mg} / \mathrm{kg}$ body weight Royal jelly. Group IV was obtained tartrazine and Cod liver oil in a dose $0.4 \mathrm{mg} / \mathrm{kg}$ body weight while the last two groups; V and VI were obtained Cod liver oil and Royal jelly separately by the above mentioned doses for 30 days.

At the end of experiment, cervical dislocation of rats and for histological studies, the liver, kidney, testis and brain tissues in addition to stomach were separately and small pieces from them were taken, fixed in neutral buffered formalin $10 \%$, dehydrated, cleared and paraffin ionized for paraffin blocks and 5 micron sections were obtained, mounted on a glass slides and stained with Hematoxylin and Eosin (H\&E) and Blue Masson's Trichrome according to Bancroft and Gamble [8].

\section{Results}

The results of histological examination in the control group as well as in groups $\mathrm{V}$ and VI showed no any histoarchitectures changes in the liver, kidney, testis, brain and stomach, suggesting that these animals were healthy and the conditions under which the experiment was conducted were proper (Figure 1). The liver parenchyma of the control group was observed very homogenous and consisting of numerous hepatic lobules that were difficult demarcated from each other's by a very thin connective tissue septa or trabeculae in between, so the hepatic lobulations were not clear (Figures $1 \mathrm{a}$ and $1 \mathrm{~b}$ ). Furthermore, they appeared hexagonal in shape and had central veins in their center. The hepatic cords were dorsally radiated from the central vein towards the periphery, the portal areas (Figures $1 \mathrm{~b}$ and $1 \mathrm{c}$ ). The major compartment of each hepatic lobule were the hepatocytes that represent about $80 \%$ of its structure and appeared irregular polygonal or polyhedral shaped cells typically with single, central, large vesicular nucleus with fine dispersed chromatins in most cases, however, some of them appeared occasionally bi-nucleated (Figure 1c). Most of hepatocytes nuclei had a central position but other located peripherally specially toward with triangular area, portal area or portal canal. The portal area is a fibro elastic connective tissue that contained branches of hepatic artery, portal vein, lymph vessels, bile duct and nerve. Moreover, the hepatic sinusoids were observed distributing in between the hepatic cords supplying the hepatocytes while bile canaliculi are located between hepatocytes rows.

Tissues sections of liver from group II, III, as well as group IV showed that, tartrazine induced sever degenerative changes which were evident in numerous hepatocytes (Figure 1). The hepatocytes were enlarged, had light and foamy cytoplasm filled with vacuoles of variable size that tended to form cystic degeneration and mostly central nuclei with proliferation of Kupffer cells (Figures $1 j-11$ and $2 f-2 i$ ). Hepatocytes necrotic changes were evident; small, pyknotic cellular nuclei with

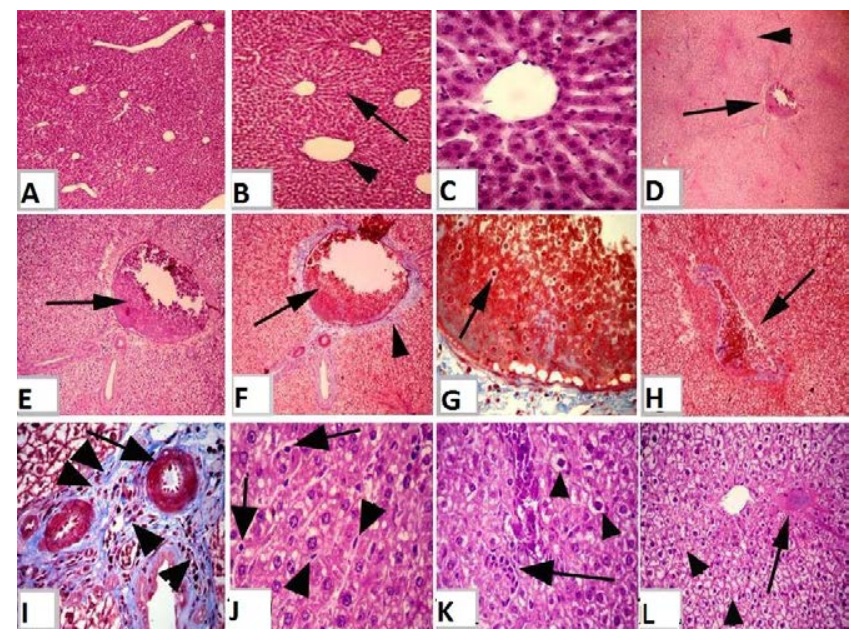

Figure 1: (a): Section of mature Westar rats liver of control group (I) showing normal, homogenous, intact hepatic parenchyma; hepatic lobules, with normal central vein. H\&E 4X:10X. (b): Section of mature Westar rats liver of control group showing normal hexagonal hepatic lobules with normal, regular radiated hepatic cords from the central vein to the peripheral of lobule (arrow), central vein (arrow head). H\&E 10X:10X. (c): Section of mature Westar rats liver of control group showing normal hepatocytes of irregular polygonal shaped cells with single, central, large vesicular nucleus. Normal and regular hepatic cords that were dorsally radiating from the central vein. H\&E 40X:10X. (d): Section of mature Westar rats liver of exposed group to tartrazine (II) showing severe steatosis, diffuse degeneration, necrosis of hepatic tissues with pale acidophilic hepatic parenchyma (arrow head) and with moderate disorganization of hepatic cords and sever congestion of portal vein (arrow). H\&E 4X:10X. (e,f): Section of mature Westar rats liver of exposed group to tartrazine (II) showing sever congestion of portal vein (arrow) with fibrous tissue proliferation in the portal areas (arrow head). e) H\&E, f) Blue Masson's Trichrome, e) 10X:10X f) 10X:10X. g): Section of mature Westar rats liver of exposed group to tartrazine (II) showing sever congestion of portal vein that overfilled with erythrocytes and some lymphocytes (arrow). Blue Masson's Trichrome 40X:10X. (h): Section of mature Westar rats liver of exposed group to tartrazine (II) showing sever congestion of portal vein (arrow) with fibrous tissue proliferation (arrow). Blue Masson's Trichrome 10X:10X. i): Section of mature Westar rats liver of exposed group to tartrazine (II) showing dilated blood vessels of the portal area (arrow) with fibrous connective tissue proliferation and leukocytes infiltrations (arrow head). Blue Masson's Trichrome 40X:10X. (j): Section of mature Westar rats liver of exposed group to tartrazine (II) showing the presence of Kupffer cells (arrow head) and lymphocytes (arrow) in between the necrotic hepatic cords. H\&E 40X:10X. (k): Section of mature Westar rats liver of exposed group to tartrazine (II) showing enlarged necrotic hepatocytes with light and foamy cytoplasm that filled with vacuoles of variable size and mostly central pyknotic nuclei (arrow head) and leukocytes infiltrations (arrow). H\&E 40X:10X. (I): Section of mature Westar rats liver of exposed group to tartrazine (II) showing wide area of necrotic hepatic parenchyma with light cytoplasm and mostly pyknotic nuclei (arrow head) and focal area of degenerated hepatocytes (arrow). H\&E 20X:10X.

condensed chromatin, lack of nucleolus and acidophilic cytoplasm were observed (Figures 2a, 2h and 2i). Mononuclear cell infiltrates were also noted in-between hepatocytes and the trabecular structure of the lobules was blurred. (Figures 1i-1k). The portal area was thickened and contained dilated vessels (Figures 1e, 1f, 1h, 1i and 2d) and thickened hyperplastic bile duct (Figures $2 \mathrm{~b}$ and $2 \mathrm{c}$ ). Most of portal veins in the portal areas were congested and overfilled with erythrocytes (Figures 1e-1h), leukocytes and in particular lymphocytes (Figure 1g). In numerous liver sections of the tartrazine group, the necrotic cells were evident after exposure to tartrazine alone with an increased density of nuclear chromatin and a very compact nuclear structure. And also, thickening in the central vein was noticed (Figure 2a). Moreover, dense collagenous connective tissue fiber proliferation and thickening around the blood vessels and bile ducts of the portal area were observed 
Citation: Ghonimi WAM, Elbaz A (2015) Histological Changes of Selected Westar Rat Tissues Following the Ingestion of Tartrazine With Special Emphasis on the Protective Effect of Royal Jelly and Cod Liveroil. J Cytol Histol 6: 346. doi:10.4172/2157-7099.1000346

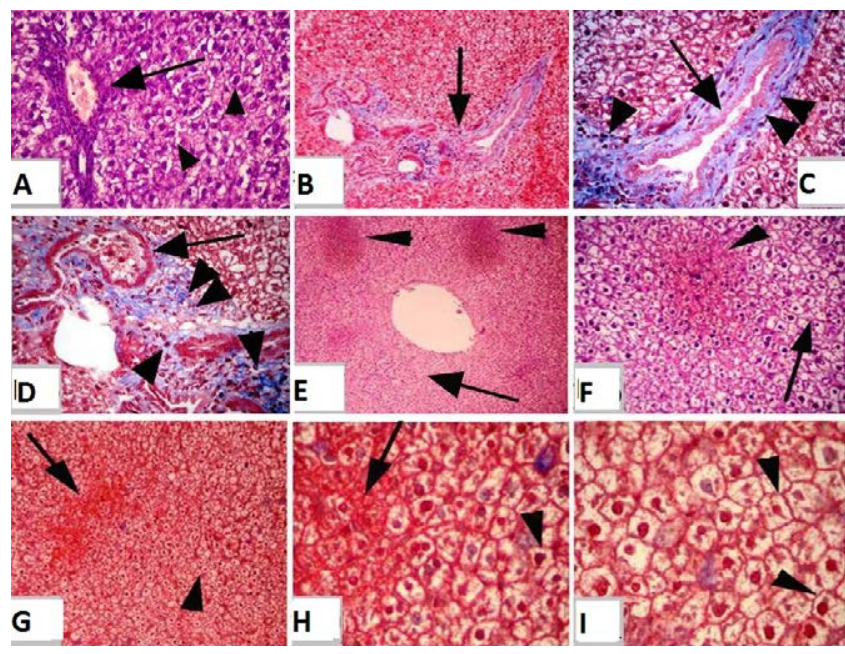

Figure 2: (a): Section of mature Westar rats liver of exposed group to tartrazine (II) showing thickening of the wall of central vein (arrow) and was surrounded with wide area of necrotic hepatocytes with pyknotic nuclei (arrow head). H\&E Obj.x20 : 10X. (b-d): Section of mature Westar rats liver of exposed group to tartrazine (II) (b): showing fibrous connective tissue proliferation in the portal areas (arrow). (c): showing thickening of bile duct (arrow), fibrous connective tissue proliferation around bile duct (double arrow head) and leukocytes infiltrations (single arrow head). (d): showing congested blood vessels (arrow), fibrous connective tissue proliferation around blood vessels (double arrow head) and leukocytes infiltrations (single arrow head).(b-d) Blue Masson's Trichrome. b) 10X : 10X c,d) Obj.x20:10X. (e, f): Section of mature Westar rats liver of exposed group to tartrazine with royal jelly (III) (e): showing small foci of normal homogenous, acidophilic hepatic tissue (arrow head) beside pale, necrotic and degenerated hepatic parenchyma (arrow). (f): higher magnification of Figure e showing the same. (e,f) H\&E e) 4X : 10X f) Obj.x20 : 10X. (g-i): Section of mature Westar rats liver of exposed group to tartrazine with cod liver oil (IV) showing the same as in group III. (g): showing foci of normal acidophilic hepatic tissue (arrow) in between necrotic and degenerated pale hepatic parenchyma (arrow head). (h): higher magnification of Figure g showing the same. (i): showing enlarged, vacuolated, pale acidophilic necrotic hepatocytes with pyknotic nucleus (arrow head). ( $\mathrm{g}-\mathrm{i}$ ) Blue Masson's Trichrome. g) 4X:10X (h,i) 4X0 : 10X.

(Figures 1e, 1f, 1h, 1I, 2b, 2c and 2d). Regarding the group III, and IV, the present investigation showed that the curative effect of both royal jelly and cod liver oil were non-significant against tartrazine toxicity. Furthermore, small foci of homogenous acidophilic hepatocytes were distributed in between wide area of necrotic cells with pale vacuolated cytoplasm and pyknotic nucleus (Figures 2e-2i).

The kidney of the control group showed no any histological changes in both cortex and medulla where, there was no degeneration of renal tubules as well as in the glomerulus (Figures $3 a$ and $3 b$ ). While there were changes occurred in the kidney of group II, where the examined sections showed degenerative changes in the renal tubules and glomeruli (Figure 3c). An enlargement of renal glomeruli, with separation and vacuolation of its lining epithelial cells as well as congestion of its blood capillaries, was observed (Figure 3d). Furthermore, hypertrophy and degeneration of the lining epithelial cells of the renal tubules (Figure 3e), with hyperemia of the medullary and cortical parts with mononuclear cell infiltrates as well as hyaline degenerative changes were found in renal tubules in all animals treated with tartrazine (Figure 3f). Mononuclear cell infiltrates were observed in some places of the medullary part of the kidney with fibrous tissue proliferation. (Figure $3 g$ ). The most advanced change after tartrazine exposure was the blood vessels dilatation that were congested and overfilled with erythrocytes in between the renal tubules (Figures $3 \mathrm{~h}$ and 3i). Generally, the histological changes in kidney cortex and medulla of group II were more serious but in group
III and IV the protective effect of cod liver oil and royal jelly were nonsignificant as in liver. While group V and VI were as normal

Sections of the testis of control group showed normal architecture of the seminiferous tubules and the interstitial tissues including Leydig cells. The seminiferous tubules appeared rounded or oval in shape and were surrounded by a thin basal Lamina (Figures $4 \mathrm{a}$ and $4 \mathrm{~b}$ ). The stratified germinal epithelium that lined the seminiferous tubules, consists of two distinct populations of cells; the proliferating, highly dividing numerous spermatogenic cells and non-dividing fewer Sertoli cells (Figures $4 \mathrm{c}$ and $4 \mathrm{~d}$ ).

Complete set of the germinal epithelial cells was present in the testis of control group. In between the tubules, the interstitial tissue had blood vessels with clusters of cells of ovoid or polygonal shape and spherical nuclei representing the leydig cells were observed as well as flat, squamous cells with flat nuclei representing the myoid cells were also noticed (Figure $4 \mathrm{~d}$ ).

All the tissue sections obtained from the testis of experimental Westar rats were not different from the control animals tissue with exception of moderate changes in the interstitial tissue of some examined sections of group II, mainly in the interstitial connective tissue not in the interstitial Leydig cells in the form of vacuolation of variable sizes, moderate edema that we can say that all the sections were essentially normal without any histological changes (Figures 4e-4l).

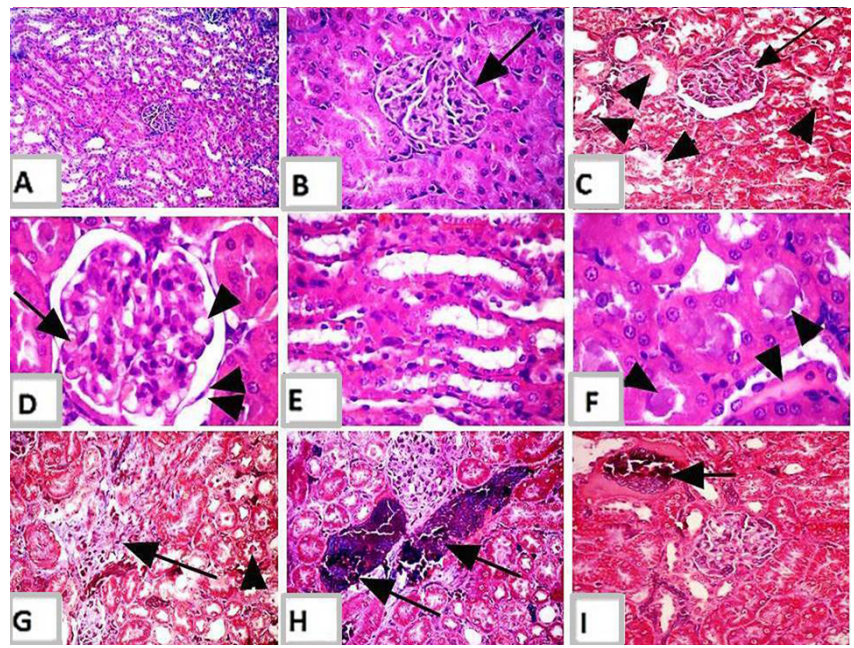

Figure 3: (a,b): Section of mature Westar rats kidney of control group (I). (a): showing normal, intact renal tubules as well as renal glomeruli. (b): higher magnification of Figure a showing the same. (a,b) H\&E a) 10X:10X b) 4X0: 10X (c): Section of mature Westar rats kidney of exposed group to tartrazine (II) showing degenerative changes in the renal tubules (arrow head) and glomeruli (arrow). Blue Masson's Trichrome Obj.x20: 10X. (d): Section of mature Westar rats kidney of exposed group to tartrazine (II) showing an enlargement of renal glomeruli with vacuolations (single arrow head) and separation of its lining and epithelial cells (double arrow head) as well as congestion of its blood capillaries (arrow). H\&E 4X0: 10X. (e): Section of mature Westar rats kidney of exposed group to tartrazine (II) showing hypertrophy and degeneration of the lining epithelial cells of the renal tubules. H\&E Obj.x20: Oc.x15. (f): Section of mature Westar rats kidney of exposed group to tartrazine (II) showing hyaline degenerative changes in the renal tubules (arrow head). H\&E 4X0: 10X (g): Section of mature Westar rats kidney of exposed group to tartrazine (II) showing fibrous connective tissue proliferation with leukocytes infiltration in between renal tubules (arrow) and hyaline degenerative changes in the renal tubules (arrow head). Blue Masson's Trichrome Obj.x20: 10X. (h,i): Section of mature Westar rats kidney of exposed group to tartrazine (II) showing blood vessels dilatation and were congested and overfilled with erythrocytes in between the renal tubules (arrow). (h,i) Blue Masson's Trichrome $(h, i) \mathrm{Obj}$. x20: $10 X$. 
Citation: Ghonimi WAM, Elbaz A (2015) Histological Changes of Selected Westar Rat Tissues Following the Ingestion of Tartrazine With Special Emphasis on the Protective Effect of Royal Jelly and Cod Liveroil. J Cytol Histol 6: 346. doi:10.4172/2157-7099.1000346

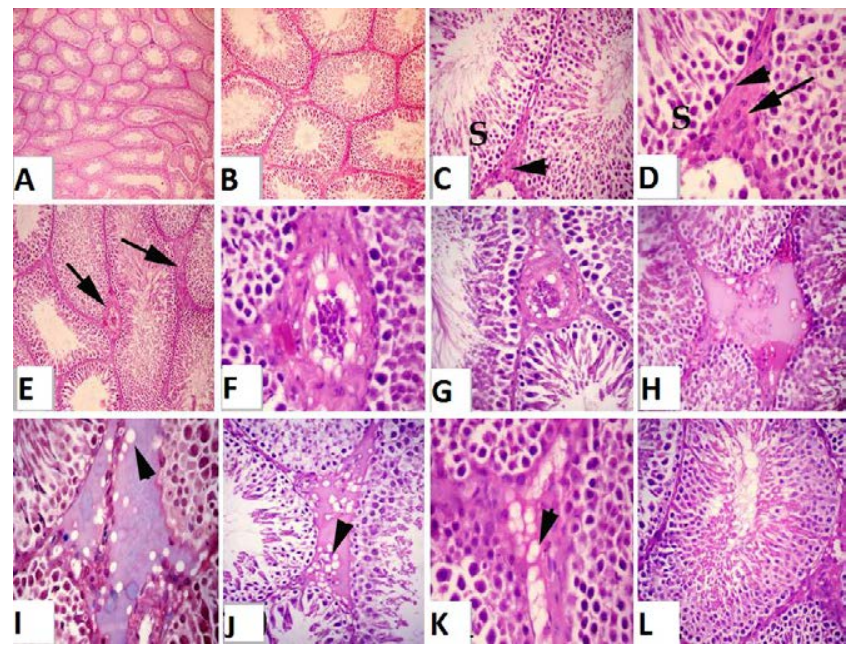

Figure 4: (a): Section of mature Westar rats testis of control group (I) showing normal, intact rounded or oval seminiferous tubules. H\&E 4X:10X. (b): Section of mature Westar rats testis of control group showing closely packed seminiferous tubules separated from each other by a considerable amoun of interstitial connective tissue. H\&E 10X:10X. (c): Section of mature Westar rats testis of control group showing normal interstitial connective tissue (arrow head) and normal; proliferating highly divided germ cells with non-divided elongated sertoli cells (S). H\&E Obj.x20: 10X. (d): Section of mature Westar rats testis of control group showing normal interstitial connective tissue with ovoid or polygonal leydig cells (arrow) and flat myoid cells (arrow head). H\&E 4X0: 10X. (e): Section of mature Westar rats testis of exposed group to tartrazine (II) showing proliferation and hyperplasia of the interstitial leydig cells (arrow). H\&E 10X : 10X. (f,g): Section of mature Westar rats testis of exposed group to tartrazine (II) showing proliferation and hyperplasia of the interstitial leydig cells with vacuolation of the interstitial CT. (f,g) H\&E f) 4X0 : 10X. g) Obj. x20: Oc.x15. (h,i): Section of mature Westar rats testis of exposed group to tartrazine with royal jelly (III) showing also proliferation and hyperplasia of the interstitial leydig cells with vacuolation of the interstitial CT. h) H\&E Obj.x20: 10X. i) 4X0: 10X Blue Masson's Trichrome. (j,k): Section of mature Westar rats testis of exposed group to tartrazine with code liver oil (IV) showing the same as exposed group to tartrazine with royal jelly. (j,k ) H\&E j) Obj.x20: 10X k) 4X0: 10X. (I): Section of mature Westar rats testis of group (V, VI) showing normal, intact seminiferous tubules with normal stratified seminiferous lining epithelium and normal interstitial connective tissue. H\&E I) Obj.x20: 10X.

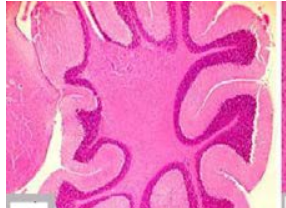

A

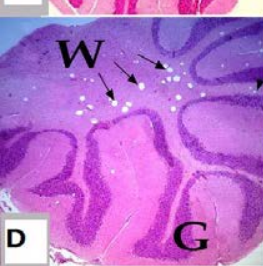

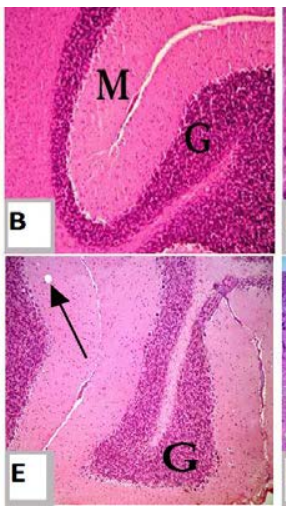

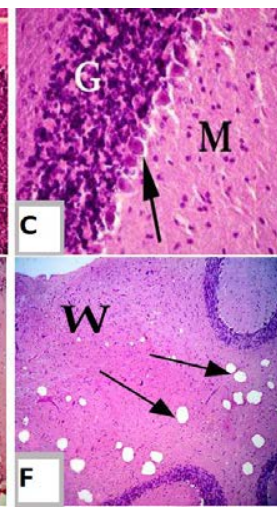

Figure 5: (a-c): Section of mature Westar rats cerebellum of control group (a): showing normal, intact cerebellar cortex and medulla. (b): showing intact molecular layer (M) and granular layer $(G)$. (c): showing normal molecular layer (M) and granular layer (G) and purkinje layer (arrow). (a-c) H\&E a) 4X:10X. b) 10X:10X. c) 4X0: 10X. (d-f): Section of mature Westar rats cerebellum of exposed group to tartrazine (II). (d): showing numerous vacuolations of variable sized in cerebellar medulla (arrow), white matter (W) and gray matter $(G)$. (e): showing slightly vacuolation in gray matter; cortex (arrow). (f): showing numerous vacuolations of variable sized in cerebellar medulla; white matter. d-f) H\&E d) 4X:10X. e,f) 10X 10X.
The cerebellar sections obtained from control group of Westar rats showed normal architecture in both cortex and medulla where three layers of cerebellar cortex, the molecular, purkinje and granular layers, appeared clear and distinct without any changes in their cells, any inflammatory cells or gliosis (Figures 5a-5c).

The main effect of tartrazine was observed in the white matter of both cerebrum and cerebellum especially medulla where the myelin sheath was more affected. Extensive myelin vacuolation within the cerebellar white matter of rats was observed. These vacuolar clefts are typically empty and represent the classic pattern of "myelin sheath splitting". Some of these vacuoles contain small amounts of poorly stained material (Figures $5 \mathrm{~d}$ and $5 \mathrm{f}$ ). While the gray matter was little affected as a very few vacuolations were observed within the cerebellar gray matter (Figure 5e).

The cerebral sections obtained from control group of Westar rats showed normal architecture in both cortex and medulla without any changes in their cells without any inflammatory cells or gliosis (Figure 6a).

The effect of tartrazine on cerebrum was typically similar to its effect on cerebellum where the main effect was observed in the white matter. Extensive myelin vacuolation within the cerebral white matter of rats was observed (Figures $6 \mathrm{c}$ and $6 \mathrm{~d}$ ). While the gray matter was little affected as few vacuolations were observed within the cerebral gray matter (Figure 6b).

However, the oligodendrocyte nuclei were microscopically normal. The oligodendrocytes with prominent perinuclear haloes, producing the typical "fried egg" were present in the cerebral gray matter. In cerebral cortex from a rat treated with tartrazine, oligodendrocytes had small, round, relatively dark nuclei and, were often in close proximity to neurons and have no any histological changes (Figure 6e). Regarding the neurons, some neurons have a large nucleus which appeared from deeply basophilc to pyknotic, and the cytoplasm became acidophilic, this means that the neurons in this way become necrotic (Figure 6e). The pervious results were relatively the same in group III and IV, no significant changes could be observed.

Sections from stomach of the control group as well as groups $\mathrm{V}$ and VI showed normal histological patterns of all tunics. The tunica mucosa was normal in its three subtonics, no degenerative changes, no leukocytic infiltration and no dilated blood vessels (Figures 7a-7c). Regarding the histological changes in the gastric mucosa of group II, we found lymphocytes infiltration in the gastric mucosa, in addition to disrupted and degenerated arrangement and architecture of the gastric mucosa, with haemorrhage in the propria and dilated and congested blood vessels (Figures 7d-7f). All tissues sections from group III and IV showed moderate and non-significant protective effects against tartrazine.

\section{Discussion}

The present study revealed that all tissue sections obtained from the stomach of experimental rats of group V and VI were not different from the control animal tissues. These results are in agreement with data reported by Amin et al. [9] who revealed that no changes were recorded in the stomach tissue of rat fed on tartrazine with Cod liver oil. Our results are also in coincidence with Moutinho et al. [10] who obtained an increase in the number of lymphocytes and eosinophils of the gastric antrum mucosa when $7.5 \mathrm{mg} / \mathrm{kg}$ bw of Tartrazine was offered in drinking water ad libitum to Westar rats from weaning to the age of twelve months. Our results are going hand to hand with data reported by Sasaki et al. [11] who studied the genotoxicity of 39 chemicals currently 
Citation: Ghonimi WAM, Elbaz A (2015) Histological Changes of Selected Westar Rat Tissues Following the Ingestion of Tartrazine With Special Emphasis on the Protective Effect of Royal Jelly and Cod Liveroil. J Cytol Histol 6: 346. doi:10.4172/2157-7099.1000346

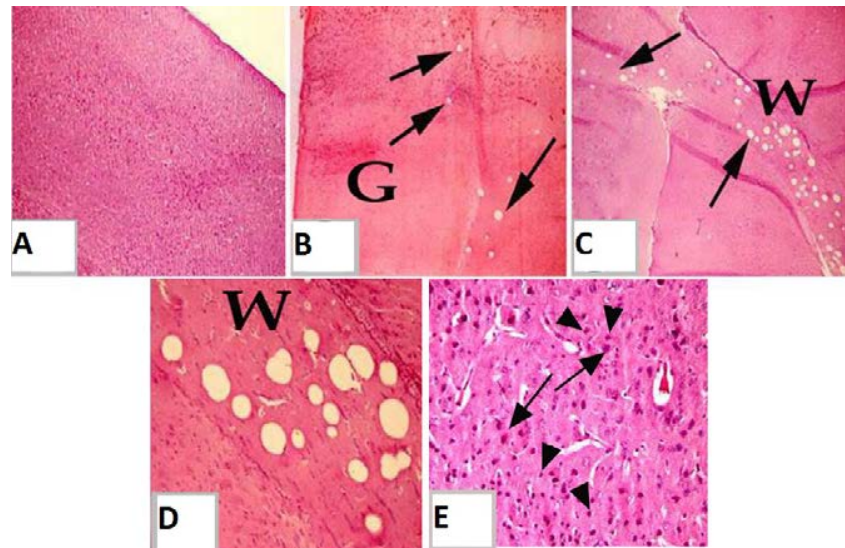

Figure 6: (a): Section of mature Westar rats cerebrum of control group (I) showing normal, intact cerebral tissue. H\&E $4 \mathrm{X}: 10 \mathrm{X}$. (b-d): Section of mature Westar rats cerebrum of exposed group to tartrazine (II) (b): showing slightly vacuolations in cerebral cortex (arrow), gray matter (G). (c): showing severe vacuolations in cerebral medulla (arrow), white matter (W). (d): higher magnification of Figure $c$ showing the same. b-d) H\&E b,c) $4 X: 10 X . d)$ Obj. x20: 10X. (e): Section of mature Westar rats cerebrum of exposed group to tartrazine (II) showing the oligodendrocyte with small, round, relatively dark nuclei (arrow head) and, were often in close proximity to neurons and have no any histological changes in the cerebral gray matter. And also, some neurons have a large nuclei which appeared from deeply basophilc to pyknotic and acidophilic cytoplasm (arrow) H\&E 4X0: 10X.

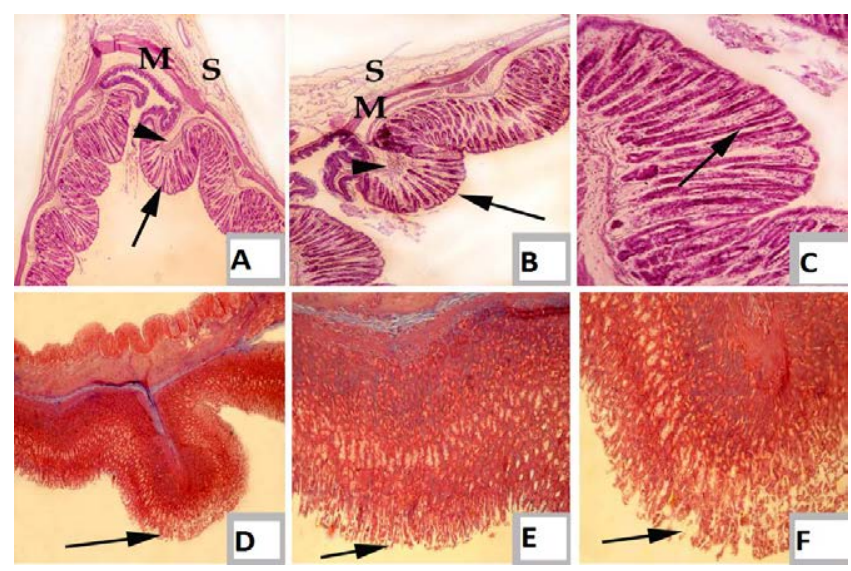

Figure 7: (a-c): Section of mature Westar rats stomach of control group (I) Figure a, b: showing normal, intact tunica mucosa (arrow), submucosa (arrow head), musculosa (M) and serosa (S). Figure c: showing normal and intact fundic gland with intact lining epithelium (arrow). (a-c) H\&E a) 4X : 10X. b) 10X:10X. c) Obj.x20: 10X. (d-f): Section of mature Westar rats stomach of exposed group to tartrazine (II) showing degenerated tunica mucosa; fundic gland and lining epithelium (arrow).d-f) Blue Masson's Trichrome d) 4X:10X. e) Obj.x20: 10X.f) Obj.x20: Oc.x15

in use as food additives. They treated groups of four male mice once orally with each additive at up to half its $\mathrm{LD}_{50}$ or the limit dose $(2000$ $\mathrm{mg} / \mathrm{kg} \mathrm{bw}$ ) and performed Comet assays on glandular stomach, colon, liver, kidney, urinary bladder, lung, brain, and bone marrow, 3 and 24 hours after treatment. He added that, tartrazine induced dose related DNA damage in the glandular stomach, colon, and/or urinary bladder and all 7 food dyes tested induced DNA damage in the gastrointestinal organs at low doses (10 or $100 \mathrm{mg} / \mathrm{kg}$ ), among them, Amaranth, Allura Red, New Coccine, and Tartrazine induced DNA damage in the colon. Tartrazine also induced DNA damage in the stomach at doses of 10 and $2000 \mathrm{mg} / \mathrm{kg}$ bw without a dose-effect relationship. The brown pigment deposition in the Küpffer cells and vacuolar degeneration of the liver in groups II, III and IV obtained in this study are in accordance with results recorded by Aboel-Zahab et al. [12] who observed brown pigment deposition in the portal tracts and Kupffer cells of the liver as well as in the interstitial tissue and renal tubular cells of the kidney. Congested blood vessels and areas of hemorrhage in both liver sections were revealed in rats of group II, III and IV receiving either Tartrazine only in a dose $500 \mathrm{mg}$ or mixture of both tartrazine with $300 \mathrm{mg} / \mathrm{kg} \mathrm{bw}$ Royal jelly and/or $0.4 \mathrm{mg} / \mathrm{kg}$ bw Code liver oil. Our results are also in coincidence with data reported by Rus et al. [13] who described changes in the liver when guinea pigs received Tartrazine in drinking water in concentrations of 1,2 and $3 \%$ for 3 weeks. For the concentration of $1 \%$, extralobular vessels and discrete perivascular edema were observed. In the external third of hepatic lobules they observed some apoptotic hepatocytes. For the concentration of $2 \%$, the liver vascular phenomena are more pronounced; capillary congestion is present in many lobules, determining a slight compression atrophy of hepatocyte cords. Hepatocytes in various stages of apoptosis were observed in small numbers within the liver lobule, the number of hepatocytes in apoptosis being greater at the lobule periphery. For $3 \%$ concentration, liver lesions are more advanced than in other concentrations.

A marked severe histological changes in the liver; congested blood vessels and areas of hemorrhage were recorded. In the early phase of fatty liver degeneration [14], vacuoles appear in the cytoplasm around the nucleus, because their lipid content is dissolved in the course of embedding. The vacuoles appear empty. As the damage to the cells progresses, the hepatocytes become swollen and a single large vacuole will occupy their entire cytoplasm, pushing aside the nucleus and making the hepatocyte signet-ring shaped are in consistency with the reporting of Rus et al. [13]. The toxic irritant substances brought to the liver by circulatory blood cause degenerative changes in the hepatic tissues, this was in line with Cameron [15]. The present study showed tubular degeneration, dilatation and vacuolation of the glomerular capillaries, intercapillary sclerosis, and atrophy of glomerulus in kidney sections from groups (II, III and IV). These changes are in consistency with the reporting of Rus et al. [13] who described changes in the kidney when guinea pigs received Tartrazine in drinking water in concentrations of 1,2 and 3\% for 3 weeks. The changes in the kidney were somewhat comparable to those of liver, meaning that there is congestion of different intensities and perivascular edema. In addition to these, abnormal glomerular filtration and glomerular or tubular stasis were observed. Renal corpuscles atrophy is present in both groups, for the group treated with Tartrazine having in addition several corpuscles and tubular necrosis. Tartrazine is transformed into aromatic amine sulfanilic acid after being metabolized by the gastrointestinal microflora [10] The formed aromatic amines can generate reactive oxygen species as part of their metabolism by interaction of these amino groups with nitrite. Reproductive studies show that tartrazine does not have effects on rats or rabbits and no adverse effects on reproductive parameters were recorded in one-generation studies at doses up to $2 \%$ in the diet $[16,17]$. So our results support the results mentioned by the pervious authors.

As recorded by Mehedi et al. [18] in mice, a gliale cell hyperplasia with congestion of blood vessels was observed in $2.5 \%$ tartrazine groups. A sub cortical edema in the brain was observed in mice treated with 1 and $2.5 \%$ tartrazine doses. Gao et al. [19] showed a decline in the activities of catalase, glutathione peroxidase (GSH-Px) and superoxide dismutase (SOD) as well as a rise in the level of malonaldehyde (MDA) in brain of tartrazine treated rats and these changes were associated with brain oxidative damage. The mechanisms might be attributed to 
Citation: Ghonimi WAM, Elbaz A (2015) Histological Changes of Selected Westar Rat Tissues Following the Ingestion of Tartrazine With Special Emphasis on the Protective Effect of Royal Jelly and Cod Liveroil. J Cytol Histol 6: 346. doi:10.4172/2157-7099.1000346

Page 6 of 6

promoting lipid peroxidation products and reactive oxygen species, inhibiting endogenous antioxidant defense enzymes and then cause brain tissue damage. Our results on brain tissues of sections from group II, III, IV were in agreement with Mehedi et al. [18] in mice and confirmed by that of Gao et al. [19]. Our results revealed that, the protective effect of both royal jelly and cod liver oil against oxidative effect of tartrazine was non-significant although amongst them [5,6] and Hünka et al. [7] investigated the antioxidant and immunostimulant effect of both cod liver oil and royal jelly. This might be due to the high dose of tartrazine in our work.

\section{Conclusion}

From our results, we can concluded that the serious effect of tartrazine toxicity are more pronounced in liver then kidney, stomach, testis and brain tissues respectively. Moreover, the curative protective effect of both royal jelly and cod liver oil are non-significant against tartrazine toxicity.

\section{References}

1. Hallagan JB, Allen DC, Borzelleca JF (1995) The safety and regulatory status of food, drug and cosmetics colour additives exempt from certification. Food Chem Toxicol 33: 515-528.

2. Walton K, Walker R, van de Sandt JJ, Castell JV, Knapp AG, et al. (1999) The application of in vitro data in the derivation of the acceptable daily intake of food additives. Food Chem Toxicol 37: 1175-1197.

3. Levine WG (1991) Metabolism of azo dyes: implication for detoxication and activation. Drug Metab Rev 23: 253-309.

4. Honohan T, Enderlin FE, Ryerson BA, Parkinson TM (1977) Intestinal absorption of polymeric derivatives of the food dyes sunset yellow and tartrazine in rats. Xenobiotica 7: 765-774.

5. Karaali A, Meydanoglu F, Eke D (1988) Studies on composition, freeze-drying and storage of Turkish royal jelly. J Apic Res 27: 182-185.

6. Bloodworth BC, Harn CS, Hock CT, Boon YO (1995) Liquid chromatographic determination of trans-10-hydroxy-2-decenoic acid content of commercial products containing royal jelly. J AOAC Int 78: 1019-1023.

7. Hünkar T, Aktan F, Ceylan A, Karasu C; Antioxidants in Diabetes-Induced
Complications (ADIC) Study Group (2002) Effects of cod liver oil on tissue antioxidant pathways in normal and streptozotocin-diabetic rats. Cell Biochem Funct 20: 297-302.

8. Bancroft JD, Gamble M (2001) Theory and practice of histological techniques (5thedn.) Churchill livingstone, New york.

9. Amin KA, Abdel Hameid $\mathrm{H}$ 2nd, Abd Elsttar AH (2010) Effect of food azo dyes tartrazine and carmoisine on biochemical parameters related to renal, hepatic function and oxidative stress biomarkers in young male rats. Food Chem Toxicol 48: 2994-2999.

10. Moutinho IL, Bertges LC, Assis RV (2007) Prolonged use of the food dye tartrazine (FD\&C yellow no 5) and its effects on the gastric mucosa of Wistar rats. Braz J Biol 67: 141-145.

11. Sasaki YUF, Kawaguchi S, Kamaya A, Ohshita M, Kabasawa K, et al. (2002 The comet assay with 8 mouse organs: results with 39 currently used food additives. Mutat Res 519: 103-119.

12. Aboel-Zahab H, el-Khyat Z, Sidhom G, Awadallah R, Abdel-al W, et al. (1997) Physiological effects of some synthetic food colouring additives on rats. Boll Chim Farm 136: 615-627.

13. Rus C, Gherman V, Miclaus A, Mihalca G, Nadas C (2004) Comparative toxicity of food dyes on liver and kidney in guinea pigs: A histopathological study. Annals of RSCB 15: 161-165.

14. Béla B, Suba Z (1999) Fatty degeneration of the liver. Introduction to Histopathology.

15. Cameron GR (1952) Pathology of the Cell, Oliver and Boyd, Edinburgh and London.

16. Elhkim M, Heraud F (2007) New considerations regarding the risk assessment on tartrazine. An updated toxicological assessment, intolerance reactions and maxiumum theoretical daily intake in France. Regulatory Tox Pharmacol 47: 308-316.

17. Tanaka T (2006) Reproductive and neurobehavioural toxicity study of tartrazine administered to mice in the diet. Food Chem Toxicol 44: 179-187.

18. Mehedi N, Mokrane N, Alami O, Ainad-Tabet S, Zaoui C, et al. (2013) A thirteen week ad libitum administration toxicity study of tartrazine in Swiss mice. African journal of biotechnology 12: 4519-4529.

19. Gao Y, Li C, Shen J, Yin H, An X, et al. (2011) Effect of food azo dye tartrazine on learning and memory functions in mice and rats, and the possible mechanisms involved. J Food Sci 76: T125-129. 